\title{
Spatial disorientation and executive dysfunction in elderly nondemented patients with Parkinson's disease
}

This article was published in the following Dove Press journal:

Neuropsychiatric Disease and Treatment

\author{
Davide M Cammisuli' \\ Simon Crowe ${ }^{2}$ \\ 'Department of Surgical, Medical, \\ Molecular and Critical Area Pathology, \\ Pisa University Medical School, Pisa, \\ Italy; ${ }^{2}$ School of Psychology and \\ Public Health, La Trobe University, \\ Melbourne, VIC, Australia
}

Objectives: Patients with Parkinson's disease (PD) present with a wide range of cognitive deficits. Cognitive impairment is recognized as an independent nonmotor aspect of the disorder and has a critical role in functional outcome and conversion into PD dementia. To date, everyday memory impairment in elderly patients with PD is underinvestigated and its relationship with executive dysfunction was not clearly explained. Our study aims at clarifying the neuropsychological pattern of everyday memory and executive deterioration in elderly patients with PD.

Methods: Forty nondemented PD patients (mean age 71.2 years; $M: F=29: 11$ ) and 30 wellmatched controls (mean age 70.7 years; $\mathrm{M}: \mathrm{F}=15: 15$ ) were assessed on everyday memory (Rivermead Behavioral Memory Test [RBMT]) and executive functioning (Frontal Assessment Battery [FAB] ) measures. Mann-Whitney $U$-tests (Bonferroni corrected) were used to compare groups on these measures and Spearman's rank correlations were performed to highlight their associations.

Results: PD patients performed worse than controls on recall for novel tasks and geographic recall (RMBT) as well as lexical fluency and mental flexibility (FAB). Particularly, spatial orientation depending on egocentric navigation seems to be altered in PD patients. The clinical group showed poorer performances than controls in mental flexibility, sensitivity to interference, and inhibitory control. Such measures were associated with immediate and delayed recall, picture recognition, prospective memory, and orientation tasks of everyday memory.

Conclusion: Executive-type difficulties and memory-type difficulties have an impact on cognitive performances of elderly patients with PD. We recommend using the RBMT and the FAB as part of routinely neuropsychological battery for assessing PD patients.

Keywords: spatial disorientation, recall impairment, executive dysfunction, elderly people, Parkinson's disease

\section{Introduction}

The main focus of research on Parkinson's disease (PD) has been devoted to motor symptoms associated with neurodegenerative disorder. However, in the last few decades, it has been increasingly recognized that nonmotor disorders may be apparent at the time of diagnosis and a variety of cognitive deficits are present in PD patients. ${ }^{1}$ Cognitive impairment may appear early in the course of PD, and it is recognized as an independent nonmotor aspect of the disorder that has a critical role in functional outcome and dementia conversion. ${ }^{2,3}$

PD was originally measured as a motor disorder by Hoehn and Yahr scale (1998), while a wider set of clinical measures and neuropsychological tests are currently used to assess PD as a complex neurodegeneration affecting sleep, ${ }^{4}$ mood, ${ }^{5}$ cognitive
Correspondence: Davide M Cammisul Department of Surgical, Medical, Molecular and Critical Area Pathology, Pisa University Medical School, Via Paradisa 2, Pisa, 56126, Italy Tel +393404123485

Fax +39058435 1745

Email d.cammisuli@med.unipi.it 
functioning, ${ }^{6}$ sensation, and perception. ${ }^{7}$ Particularly, sensory deficits may include visual, vestibular, and proprioceptive information processing, all of which are critical for spatial orientation. $^{7}$

In $\mathrm{PD}$, there is a spectrum of cognitive deterioration, ranging from mild cognitive impairment (MCI) to PD dementia (PDD). MCI is common in nondemented PD patients (mean prevalence, $27 \%$; range, $19 \%-38 \%$ ) and may be a dementia forerunner. ${ }^{8,9} \mathrm{MCI}$ due to $\mathrm{PD}$ has received increased attention in the last years, including validation studies for diagnostic criteria. $^{10,11}$

With regard to memory processes, there is evidence that PD patients perform worse than controls in a series of memory tasks, such as recognition, recall, and prospective memory ones, ${ }^{12}$ but only two investigations including nondemented PD patients evaluated everyday memory deficits by appropriate and ecologically valid memory tests, such as the Rivermead Behavioral Memory Test (RBMT), ${ }^{13}$ focusing on tasks hypothesized to serve as analogs for everyday memory skills.

Katai $^{14}$ showed that PD patients scored significantly lower on the RBMT than controls in event-based prospective memory tasks (ie, appointment and hidden personal belonging RBMT subtests) requiring attentional control. Furthermore, performances on everyday memory testing were significantly associated with cognitive flexibility and verbal fluency. More recently, no alteration of immediate and delayed recall on novel subtests was found by Eygelshoven et $\mathrm{al}^{15}$ in a larger sample of PD patients compared with normal subjects.

To date, everyday memory impairment accompanying PD in elderly patients is currently underinvestigated, probably because of more overt cognitive impairments associated with the disease, such as attention/working memory, visuospatial skills, language, prospective memory, and executive deficits. ${ }^{16}$ Moreover, studies mainly focused on younger population, drugs naïve patients, or early onset PD, and they did not take into account advanced phases, in which daily cognitive difficulties became relevant, functional autonomy decreases, and neuropsychiatric symptoms may be apparent. ${ }^{17}$

Our study aims at clarifying the neuropsychological pattern of everyday memory and executive deterioration in elderly patients with PD, by specifically depicting their neuropsychological profile.

\section{Materials and methods Subjects}

A mixed group of 40 nondemented patients with idiopathic PD (30 with normal cognition and 10 with MCI) and 30 controls constituted the sample of the study. Participants were con- secutively recruited from September 2013 until January 2018. PD patients were recruited by the Neurology Service of Felice Lotti Hospital in Pontedera (Pisa, Italy) and selected on the basis of patient's medical history, an examination of clinical signs and symptoms, a neurological and physical examination, and laboratory tests. Inclusion criteria were diagnosis of PD according to Gelb et al criteria ${ }^{18}$ and 5 years or higher education. Exclusion criteria were presence of dementia, severe apathy, serious ongoing systemic and psychiatric diseases, cardiovascular disease, and hepatic disease. MRI of the brain and DatScan were used to help rule out other disorders. PD patients were treated with doses of carbidopa/levodopa (25/100 mg) three times a day. Control group consisted of community-dwelling individuals from Valdera and Versilia areas of Tuscany (Italy) without history of neurological disease, psychiatric disease, and relevant medical conditions.

All the participants provided a written informed consent before clinical and neuropsychological assessment. The study protocol was approved by the Ethic Committee of Area Vasta Nord Ovest (Pisa, Italy).

\section{Assessment}

The two groups were assessed by a neuropsychological battery, including an overall cognitive screening and measures of everyday memory and executive functions.

Milan overall dementia assessment (MODA) ${ }^{19}$ : it is an overall cognitive screening test. It is used to evaluate a suspect of dementia in people reporting cognitive changes. It consists of three sections: orientation scale (ie, temporal, spatial, personal, and family orientation), autonomy scale (ie, walking, dressing, personal hygiene, sphincter control, and nutrition), and neuropsychology tests (ie, reversal learning, attentive matrices, verbal intelligence, memory of prose, semantic verbal fluency, token test, digital gnosis, constructive apraxia, and Street completion test). It takes about 1 hour to be administered. The raw score is the sum of the three scales: orientation scale score ( $\max 35$ points), autonomy scale score ( $\max 15$ points), and neuropsychological tests score (max 50 points). It is corrected for age and education (ie, MODA total score). The total score ranges from 0 to 100 . A score below 85.5 indicates a pathological condition, a score between 85.5 and 89.0 is considered borderline (thus a definitive clinical judgment is not allowed), and a score above 89.0 is considered to be normal.

Activities of daily living (ADL) $)^{20}$ and Instrumental $\mathrm{ADL}^{21}$ were used to evaluate functional impairment in both clinical and non-clinical group. The severity of clinical symptoms in the PD group and motor status were further 
assessed by Hoehn and Yahr (H\&Y) scale ${ }^{22}$ and unified Parkinson's disease rating scale, part III (UPDRS-III), ${ }^{23}$ respectively. Depressive symptoms in PD patients were also investigated by geriatric depression scale-30 (GDS-30) item. ${ }^{24}$

RBMT $^{25}$ : it represents an ecologically valid tool, which gives information on everyday memory problems occurring in daily life of people. It is also useful for the evaluation of patients' memory status after rehabilitation or cognitive trainings. The test has four parallel forms (A, B, C, D) that are able to reduce learning effect on retest. It consists of the following subtests: first and second names - delayed recall; belongings - delayed recall; appointment - delayed recall; picture recognition - delayed recall; novel - immediate and delayed recall; face recognition - delayed recall; route learning-immediate and delayed recall; message-immediate and delayed recall; orientation; date. It takes approximately 30-40 minutes to be administered. Each subtest gives two scores, each of one is summed up with those of the other subtests to compose profile score (PS) (ranging from 0 to 24) and screening score (SS) (ranging from 0 to 12). The SS offers an estimation of memory deficit: 12-9 (normal), 8-6 (suspect slight deficit), 5-3 (suspect severe deficit), and 2-0 (suspect very severe deficit). The PS is converted into a standardized PS (SPP). The RBMT has got high inter-rater reliability and good intertest reliability. ${ }^{25}$

Frontal Assessment Battery (FAB $)^{26}$ is a brief neuropsychological tool aiming at assessing executive functions at the bedside. It consists of six subtests, exploring abilities related to frontal domains: conceptualization (ie, similarities task), mental flexibility (ie, lexical fluency task), motor programming (ie, Luria's motor series), sensitivity to interference (ie, conflicting instructions task), inhibitory control (ie, go-no-go task), and environmental autonomy (ie, prehension behavior). It takes approximately $5-10$ minutes to be administered. Each subtest gives a score ranging from 0 to 3 , according to the quality of the performances. The raw scores obtained were corrected for age and education. ${ }^{27}$ The FAB showed good concurrent and discriminant validity and high internal consistency; test-retest and inter-rater reliability were fairly good. ${ }^{28}$

\section{Statistics}

Independent sample $t$-tests compared sociodemographic characteristics of groups, while a Pearson's chi-squared test was used for categorical variables. Statistical Package for the Social Sciences (SPSS) 23.0 software (SPSS Inc., Chicago, IL, USA) was used for data analysis.

Preliminary analysis based on the Kolmogorov-Smirnov test showed that data were non-Gaussian distributed. Thus, nonparametric tests (ie, Mann-Whitney $U$ tests) were used to compare groups on everyday memory tasks and frontal domains measures. A $P$-value $<0.05$ (Bonferroni corrected) was set to reach significance. Moreover, Spearman's correlations were performed between everyday memory and frontal domains measures in PD patients, and Pearson's correlations were performed between RBMT Standardized Profile Score (SPS)/FAB total score and GDS score.

\section{Results}

Descriptive analysis was first reported (Table 1). Most PD patients were in H\&Y stage 2 of the disease, which meant that they had mild bilateral PD signs and recovery of balance on the pull test. ${ }^{29}$ UPDRS-III score confirmed a mild-tomoderate motor impairment of the clinical sample.

The two study groups were well-matched with respect to age, education, sex ratio, and performance on global cognitive assessment measures (Table 1), except for the MODA autonomy scale score $(P=0.003)$. Spearman's correlations showed that PD disease severity was not associated with any performance on everyday memory and executive testing in the clinical group. Furthermore, Pearson's correlations showed that depression was not associated with both FAB total score and RBMT SPS score.

PD patients obtained a RBMT PS lower than controls (49.7 \pm 9.9 vs $57.7 \pm 13.1$ ). RMBT SS reported the following percentages: $22.5 \%$ of patients with normal performance, $22.5 \%$ of patients with a suspect slight deficit, $47.5 \%$ of patients with suspect severe deficit, and $7.5 \%$ of patients with suspect very severe deficit. Thus, $77.5 \%$ of PD patients showed a suspect memory deficit, from slight to very severe.

Significantly, PD patients performed worse than controls on total RBMT SPS $(P=0.000)$, route immediate recall SPS $(P=0.001)$, route delayed recall SPS $(P=0.000)$, and novel delayed recall task SPS $(P=0.000)$ (Bonferroni corrected) (Table 2, Figure 1).

Furthermore, PD patients reported lower score than controls on FAB total score $(P=0.000)$, lexical fluency $(P=0.006)$, Luria's motor series $(P=0.001)$, and go-no-go $(P=0.000)$ (Bonferroni corrected) (Table 3, Figure 1).

In the PD group, FAB total score was positively associated with RMBT SPS, novel task - immediate recall, picture recognition, appointment, and hidden personal belonging subtests (refer to Table 4 for comparison).

Lexical fluency FAB subtest was positively associated with novel task - immediate recall and highly associated with appointment $(P<0.01)$. Luria's motor series was positively associated with novel task - immediate recall, picture recognition, message (delayed recall), and hidden personal 
Table I Sociodemographic characteristics of the two samples

\begin{tabular}{llll}
\hline & PD patients (n=40), & Controls (n=30), \\
& M \pm SD, ratio, median & M \pm SD & \\
\hline Age (years) & $71.2 \pm 7.8$ & $70.7 \pm 7.8$ & 0.625 \\
Education (years) & $7.4 \pm 3.8$ & $8 \pm 3.3$ & 0.467 \\
Sex (M:F) & $29: 11$ & $15: 15$ & 0.080 \\
PD duration (years) & $3.3 \pm 2.8$ & - & - \\
MODA temporal orientation & $9.9 \pm 0.1$ & $9.9 \pm 0.2$ & 0.699 \\
MODA spatial orientation & $2.9 \pm 0.3$ & $3 \pm 0.01$ & 0.545 \\
MODA personal orientation & $9.9 \pm 0.2$ & 10 & 0.284 \\
MODA family orientation & $11.5 \pm 0.8$ & $11.9 \pm 0.1$ & 0.064 \\
MODA autonomy scale & $14.5 \pm 0.9$ & 15 & 0.003 \\
MODA neuropsychological tests & $42.4 \pm 3.6$ & $43.6 \pm 2.9$ & 0.372 \\
MODA total score & $91.2 \pm 4.6$ & $93.3 \pm 3.1$ & 0.299 \\
ADL & $0.9 \pm 0.1$ & 1 & 0.549 \\
IADL & $0.9 \pm 0.1$ & 1 & 0.286 \\
H\&Y scale & -2 & - & - \\
UPDRS-III & $17.8 \pm 10.1$ & - & - \\
GDS & $9.7 \pm 7.1$ & & \\
\hline Note: Signf & & &
\end{tabular}

Note: Significant $P$-value is shown in bold.

Abbreviations: -, not applicable; ADL, activities of daily living; GDS, geriatric depression scale; H\&Y, Hoehn and Yahr scale; IADL, instrumental activities of daily living; MODA, Milan overall dementia assessment; PD, Parkinson's disease; UPDRS-III, unified Parkinson's disease rating scale, part III.

belonging, and highly associated with RMBT SPS $(P<0.01)$. Finally, go-no-go FAB was associated to RBMT SPS, picture recognition, orientation, appointment, novel task - delayed recall, and hidden personal belonging.

\section{Discussion}

We found that nondemented PD patients performed significantly worse than controls on novel task recall, geographic recall, lexical fluency, and mental flexibility in addition to autonomy decrease. People with PD experience functional limitations early in the course of the disease. According to the literature, ${ }^{30}$ they report difficulties with some daily activities

Table 2 Groups comparison on RBMT and RBMT subtests SPS

\begin{tabular}{|c|c|c|c|}
\hline RBMT & $\begin{array}{l}\text { PD patients } \\
\text { (median) }\end{array}$ & $\begin{array}{l}\text { Controls } \\
\text { (median) }\end{array}$ & $P$-values* \\
\hline RBMT & 14.5 & 20 & 0.000 \\
\hline Novel task - immediate recall & 1 & 2 & 0.034 \\
\hline Picture recognition & 2 & 2 & 0.033 \\
\hline Route - immediate recall & 2 & 2 & 0.001 \\
\hline Face recognition & 1.5 & 2 & 0.024 \\
\hline Orientation & 2 & 2 & 0.021 \\
\hline Appointment & 1 & 2 & 0.025 \\
\hline Novel task - delayed recall & I & 2 & 0.000 \\
\hline Route - delayed recall & 1.5 & 2 & 0.000 \\
\hline Message - delayed recall & 2 & 2 & 0.034 \\
\hline $\begin{array}{l}\text { First and second name - } \\
\text { delayed recall }\end{array}$ & 0 & 0 & 0.081 \\
\hline Hidden personal belonging & I & 2 & 0.371 \\
\hline
\end{tabular}

Notes: *Bonferroni corrected. Significant $P$-values are shown in bold.

Abbreviations: PD, Parkinson's disease; RBMT, Rivermead Behavioral Memory Test; SPS, Standardized Profile Score. involving autonomy, and cognitive dysfunction is thought to be an important contributor to these changes. The result on MODA autonomy scale was not corroborated by the comparison on ADL between clinical and nonclinical groups. This may be due to the fact that MODA autonomy scale evaluates similar variables of ALD scale but it gives more weight to motor efficiency that is mild to moderate impaired in PD patients. In $\mathrm{PD}$, cognitive impairment has a great impact on quality of life and is associated with early mortality. ${ }^{31}$ Low performances on cognitive tests in nondemented PD patients is associated to an increased risk of developing dementia. ${ }^{32}$ Beyond cognitive and motor symptoms severity, risk factors also include older age. ${ }^{33}$

To our best knowledge, the main result of our study was the demonstration of spatial orientation deficits related to everyday tasks in PD patients. Remarkably, the RBMT route subtasks requiring walking in a room by respecting five different steps previously shown by the examiner that have to be reproduced in an immediate (after the presentation) and delayed condition (after interference tasks) by the examinee imply a sense of navigational guidance within a restricted environment. Spatial navigation, or the ability of maintaining a trajectory from one place to another, gradually declines in the course of physiological aging, ${ }^{34}$ and spatial disorientation represents a pathological feature of some neurodegenerative conditions, such as Alzheimer's disease. ${ }^{35}$ Spatial navigation is supported by multiple parallel representations (ie, allocentric, egocentric, and path integration) that can be 


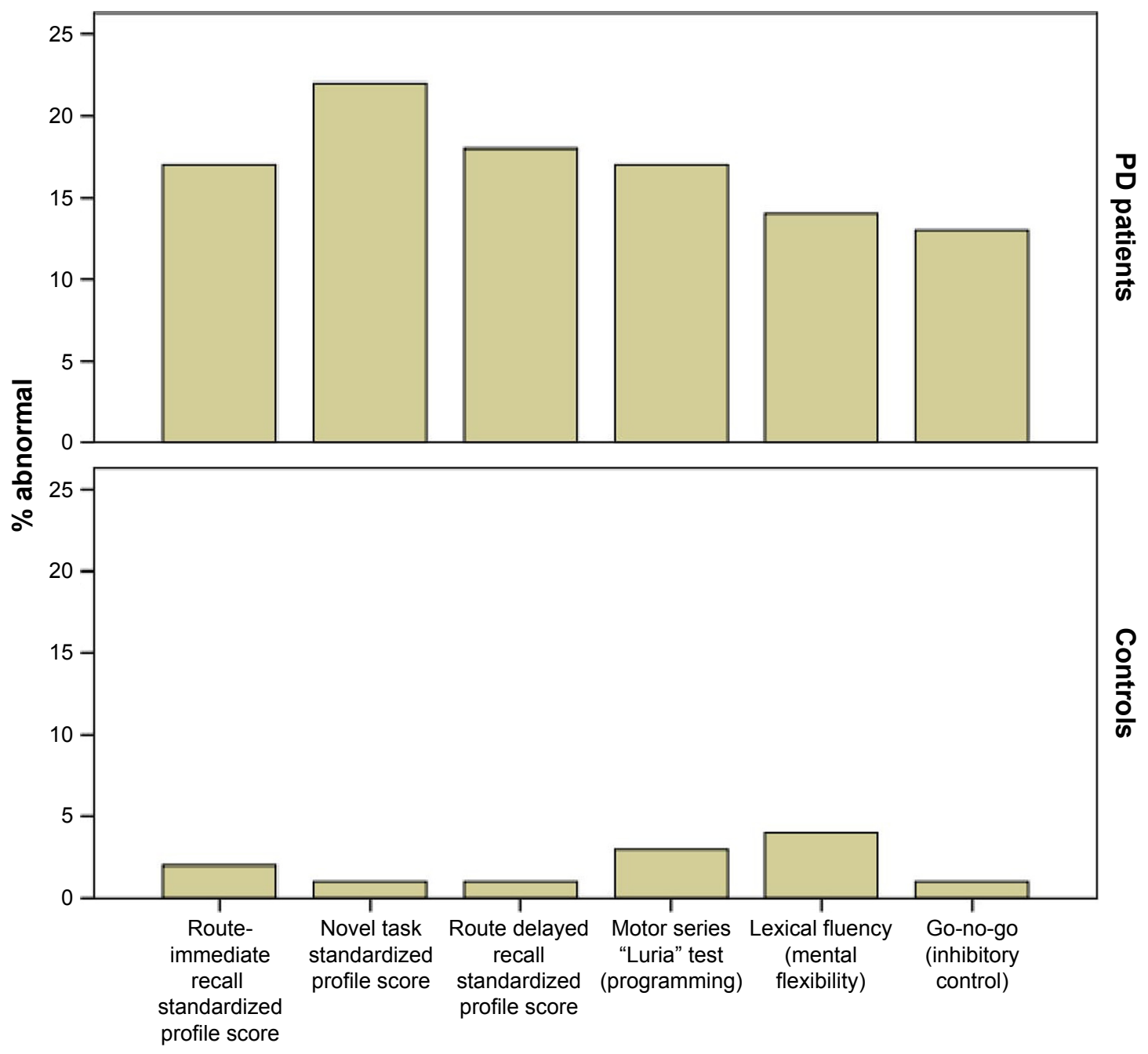

Figure I Percentage of abnormal scores on RBMT and FAB subtests.

Abbreviations: FAB, Frontal Assessment Battery; PD, Parkinson's disease; RBMT, Rivermead Behavioral Memory Test.

dissociated behaviorally and in terms of their neural basis, with the hippocampus and medial temporal lobe providing allocentric environmental representations, the parietal lobe egocentric representations, and retrosplenial cortex and parietal-occipital sulcus allowing both type of representations to interact. ${ }^{36}$ Moreover, hippocampal and striatal

Table 3 Groups comparison on FAB

\begin{tabular}{llll}
\hline & $\begin{array}{l}\text { PD patients } \\
\text { (median) }\end{array}$ & $\begin{array}{l}\text { Controls } \\
\text { (median) }\end{array}$ & P-values* \\
\hline FAB total score & 13.9 & 16.8 & $\mathbf{0 . 0 0 0}$ \\
Similarities & 2 & 2.5 & 0.059 \\
Lexical fluency & 2 & 3 & $\mathbf{0 . 0 0 6}$ \\
Prehension behavior & 3 & 3 & 0.732 \\
Luria's motor series & 2 & 3 & $\mathbf{0 . 0 0 1}$ \\
Conflicting instruction & 3 & 3 & 0.025 \\
Go-no-go & 2 & 3 & $\mathbf{0 . 0 0 0}$ \\
\hline
\end{tabular}

Notes: *Bonferroni corrected. Significant $P$-values are shown in bold. Abbreviations: FAB, Frontal Assessment Battery; PD, Parkinson's disease. system processes different aspects of environmental layout that are boundaries and local landmarks, respectively. ${ }^{37,38}$ According to these evidences, we suggest that elderly PD patients might present with an altered spatial orientation more related to egocentric than allocentric representations. Parietal-mediated perception of visual space is affected in PD and may explain patients' difficulties with egocentric spatial navigation beyond motor dysfunction..$^{39}$ Notably, such a kind of spatial orientation deficit was reported both in the immediate and delayed conditions of the route recall subtests and not influenced by executive abilities, as confirmed by the absence of correlations among these RBMT subtests and FAB total score and subtests scores. Thus, it represents a particular feature of $\mathrm{PD}$ patients' neuropsychological profile. Deficits we found are related to self-oriented navigation for which involvement of the posterior parietal areas is due $e^{40,41}$ and not to a distorted allocentric navigation accounting for 
Table 4 Correlations between RMBT total and subtests SPS and FAB total score and subtests scores in PD group

\begin{tabular}{|c|c|c|c|c|c|c|c|}
\hline & FAB & Similarities & $\begin{array}{l}\text { Lexical } \\
\text { fluency }\end{array}$ & $\begin{array}{l}\text { Prehension } \\
\text { behavior }\end{array}$ & $\begin{array}{l}\text { Luria's motor } \\
\text { series }\end{array}$ & $\begin{array}{l}\text { Conflicting } \\
\text { instruction }\end{array}$ & Go-no-go \\
\hline RMBT & $0.384^{*}$ & 0.141 & 0.288 & -0.106 & $0.55 I^{* *}$ & 0.131 & $0.377 *$ \\
\hline Novel task - immediate recall & $0.34 I *$ & 0.103 & $0.349 *$ & -0.233 & $0.322 *$ & 0.073 & 0.233 \\
\hline Picture recognition & $0.350 *$ & -0.001 & 0.111 & -0.025 & $0.334^{*}$ & 0.190 & $0.393 *$ \\
\hline Route - immediate recall & 0.120 & 0.091 & 0.252 & 0.053 & 0.107 & -0.023 & -0.076 \\
\hline Face recognition & -0.013 & -0.037 & 0.119 & -0.240 & 0.187 & 0.042 & -0.168 \\
\hline Orientation & 0.078 & 0.087 & 0.148 & -0.176 & -0.065 & 0.182 & $0.333 *$ \\
\hline Appointment & $0.345^{*}$ & -0.017 & $0.467 * *$ & 0.252 & 0.199 & -0.026 & $0.372 *$ \\
\hline Novel task - delayed recall & 0.301 & 0.231 & 0.313 & -0.012 & 0.254 & 0.070 & $0.386 *$ \\
\hline Route - delayed recall & 0.230 & 0.073 & 0.169 & -0.213 & 0.119 & 0.027 & 0.046 \\
\hline Message - delayed recall & 0.098 & 0.253 & 0.039 & 0.092 & $0.391 *$ & 0.186 & -0.004 \\
\hline First and second name - delayed recall & 0.036 & 0.097 & 0.187 & 0.111 & 0.051 & -0.261 & 0.095 \\
\hline Hidden personal belonging & $0.377^{*}$ & 0.265 & -0.212 & -0.232 & $0.392 *$ & 0.099 & $0.377^{*}$ \\
\hline
\end{tabular}

Notes: $* p<0.05 ; * * P<0.01$.

Abbreviations: FAB, Frontal Assessment Battery; PD, Parkinson's disease; RBMT, Rivermead Behavioral Memory Test; SPS, Standardized Profile Score.

the loss of dopaminergic innervation to the dorsal striatum that is implicated in cognitive tasks requiring executive abilities. ${ }^{42}$ Results on RBMT novel task showed that PD patients perform poorer than controls, too. As commonly recognized ${ }^{43}$ PD patients demonstrate impaired free recall due to disruption of strategies for search and retrieve of information, presumably due to an executive dysfunction in this case. This finding is consistent with the fact that people with frontal lobe dysfunction report poor performances in recall tasks, especially in delayed ones after interfering activities. ${ }^{44}$ Although causality cannot be directly inferred, a relation between executive functioning and verbal and visual memory is evident, as shown by the correlations between RBMT novel recall (immediate and delayed) with mental flexibility, sensitivity to interference, and inhibitory control. Researchers should consider this association when interpreting reduced performances of these two cognitive domains. Finally, our result is different from that of Eygelshoven et al, ${ }^{15}$ probably due to different cognitive phenotype of PD patients involved, mainly reporting an attention/psychomotor speed deficit. We recommend including everyday memory measures with high ecological validity in PD cognitive assessment by means of tasks that mimic ordinary challenges, given that PD routinely experience memory failures in day-to-day demands. The RMBT may be part of routinely cognitive assessment process of psychogeriatric settings that may inform clinicians about detection and monitoring of memory changes in older adults and about evaluation of rehabilitation and treatment outcomes.

The second result of our study was the association between some FAB and RBMT scores. Weakness of frontal efficiency evidenced by the scores obtained by PD patients on $\mathrm{FAB}$ were in line with many investigations reporting frontal lobe involvement in PD. ${ }^{45-47} \mathrm{FAB}$ is sensitive to frontal lobe involvement in $\mathrm{PD}$, as reflected by lower gray matter density in prefrontal areas. ${ }^{48}$ Specifically, deficit of lexical fluency, motor programming, and inhibitory control clearly depict PD cognitive disorder, mainly characterizing by the inability to build up self-generated cognitive strategies to retrieve semantic memory information, organization, maintenance and execution of concatenated actions (ie, Luria's series, "fist-palm-edge"), and difficulty to inhibit inappropriate responses, respectively. Correlations we found confirm that such executive abilities play a critical role in everyday memory performance, especially influencing immediate and delayed recall, picture recognition, prospective memory, and orientation. Neuroanatomical correlates of executive functions mainly include ventromedial prefrontal cortex, dorsolateral prefrontal cortex, and anterior cingulate cortex. The ventromedial prefrontal cortex is predominantly associated with deficits in emotional/social aspects of executive functions, whereas dorsolateral prefrontal and anterior cingulate cortices are predominantly associated with deficits in cognitive aspects of executive functions. ${ }^{49}$ In line with our findings, brain lesions on the last two frontal areas may impair free recall and recognition with a different extent and affect source memory and temporal order. ${ }^{50}$

A potential limitation of the study concerned the use of tasks involving motor responses, such as Luria's motor series and go-no-go FAB subtests, in which PD patients showed a declined performance. ${ }^{51}$ However, scores of these subtests did not take into account motor slowing or reducing but focused on correct execution of the examiner's command, thus minimizing motor demands. Visual defects in complex tasks 
including the ability to judge distances, visual field sensitivity, and visual processing speed should take into consideration when PD patients are assessed as well as visual-constructive skills. ${ }^{52}$ Results on RBMT route immediate and delayed recall should be read with caution, given negative implications depending on motor impairment (ie, muscle power and mobility) of PD patients upon spatial orientation performances, even if spatial orientation has been found not to be influenced, for example, by balance control in the last phases of PD. ${ }^{53}$ Furthermore, the use of other standardized memory tests should be considered to specifically evaluate the suspect memory deficit reported by the $77.5 \%$ of PD patients.

Another constraint of our study consists of spatial performance assessment. On the basis of our findings, further investigations should involve visuospatial short-term memory assessment (eg, Corsi blocks test) as well as spatial planning assessment (eg, Elithorn perceptual maze test), in order to evaluate the potential role of visuospatial sketchpad capacity and perceptual/executive integrity in PD patients, with the final aim of pointing out an egocentric navigation deficit more accurately. Finally, dopaminergic medication plays a critical role for cognition in PD. It has been shown to have different effects, globally enhancing executive functions while the effects of dopamine replacement on memory retrieval remains unclear. ${ }^{54}$

\section{Conclusion}

Our study pointed out a spatial cognition deficit that may affect egocentric navigational guidance in everyday environment along with distinct executive deficits. Memory capacity needed to adequately manage one's day-to-day living environment presumably calls on a combination of longterm declarative memory and executive control efficiency, especially relevant in elderly people. Particularly, spatial disorientation may represent a specific characteristic of the neuropsychological profile of PD patients in later stages, differently from those in early stages of Alzheimer's disease, in which spatial disorientation denotes a typical emerging symptom alongside with episodic memory impairment. ${ }^{55}$

Cognitive functioning represents a key area in PD. Executive-type and memory-types difficulties may influence the ADL of elderly PD patients, with negative implications for their quality of life. The progressive deterioration of cognitive functioning improves caregiver burden and may be present along with neuropsychiatric symptoms. ${ }^{56}$ The RMBT and FAB should be part of the neuropsychological battery in assessing PD patients: these tests can be administered quickly without caregiver assistance and examinees do not experience frustration unlike in traditional tests. ${ }^{57}$ Further studies will be needed to see whether cognitive rehabilitation can improve autonomy in PD patients.

\section{Acknowledgments}

The authors gratefully thank clinicians from the Neurology Service (Felice Lotti Hospital of Pontedera, Pisa, Italy) for inviting patients to be further examined at the Neuropsychology Clinic of Pontedera (Pisa, Italy) and Marco Timpano Sportiello, Emanuela Castro, Baroncini Matteo, Michele Gnoffo, Stefania Tocchini, and her colleagues for their help in collecting neuropsychological data of PD patients and controls from Valdera (Pisa, Italy) area.

\section{Author contributions}

DMC provided substantial contributions to conception and design, acquisition of data, analysis, interpretation of data, and drafting the article. SC provided substantial contribution for data analysis and results interpretation and revised the article for intellectual content. Both authors approved the final version.

\section{Disclosure}

DMC has received research funding from the Michael J. Fox Foundation for Parkinson's Research as a member of the Italian Network of Parkinson's Disease-Mild Cognitive Impairment for Pisa site (Italy). The authors report no other conflicts of interest in this work.

\section{References}

1. Goldman JG, Litvan I. Mild cognitive impairment in Parkinson's disease. Min Med. 2011;102(6):441-459.

2. Kehagia AA, Barker RA, Robbins TW. Neuropsychological and clinical heterogeneity of cognitive impairment and dementia in patients with Parkinson's disease. Lancet Neurol. 2010;9(12):1200-1213.

3. Pigott K, Rick J, Xie SX, et al. Longitudinal study of normal cognition in Parkinson disease. Neurology. 2015;85(15):1276-1282.

4. Chahine LM, Amara AW, Videnovic A. A systematic review of the literature on disorders of sleep and wakefulness in Parkinson's disease from 2005 to 2015. Sleep Med Rev. 2017;35:33-50.

5. Balestrino R, Martinez-Martin P. Neuropsychiatric symptoms, behavioural disorders, and quality of life in Parkinson's disease. J Neurol Sci. 2017;373:173-178.

6. Schrag A, Weintraub D, Schott JM. Cognitive decline before diagnosis of Parkinson's disease - authors' reply. Lancet Neurol. 2017; 16(4):262.

7. Barnett-Cowan M, Dyde RT, Fox SH, et al. Multisensory determinants of orientation perception in Parkinson's disease. Neuroscience. 2010;167(4):1138-1150.

8. Litvan I, Aarsland D, Adler CH, et al. MDS Task Force on mild cognitive impairment in Parkinson's disease: critical review of PD-MCI. Mov Disord. 2011;26(10):1814-1824.

9. Litvan I, Goldman JG, Tröster AI, et al. Diagnostic criteria for mild cognitive impairment in Parkinson's disease: Movement Disorder Society Task Force guidelines. Mov Disord. 2012;27(3):349-356. 
10. Geurtsen GJ, Hoogland J, Goldman JG, et al. Parkinson's disease mild cognitive impairment: application and validation of the criteria. J Parkinsons Dis. 2014;4(2):131-137.

11. Hoogland J, Boel JA, de Bie RMA, et al. Mild cognitive impairment as a risk factor for Parkinson's disease dementia. Mov Disord. 2017; 32(7):1056-1065

12. Whittington CJ, Podd J, Stewart-Williams S. Memory deficits in Parkinson's disease. J Clin Exp Neuropsychol. 2006;28(5):738-754.

13. Wilson B, Cockburn J, Baddeley A, Hiorns R. The development and validation of a test battery for detecting and monitoring everyday memory problems. J Clin Exp Neuropsychol. 1989;11(6):855-870.

14. Katai S. Everyday memory impairment in Parkinson's disease. Clin Neurol. 1999;39(3):913-919.

15. Eygelshoven S, van den Hout A, Tucha L, et al. Are non-demented patients with Parkinson's disease able to decide about their own treatment? Parkinsonism Relat Disord. 2017;38:48-53.

16. Trojano L, Papagno C. Cognitive and behavioral disorders in Parkinson's disease: an update. II: behavioral disorders. Neurol Sci. 2018; 39(1):1-9.

17. Martínez-Martín P, Benito-León J, Alonso F, et al. Quality of life of caregivers in Parkinson's disease. Qual Life Res. 2005;14(2):463-472.

18. Gelb DJ, Oliver E, Gilman S. Diagnostic criteria for Parkinson disease. Arch Neurol. 1999;56(1):33-39.

19. Brazzelli M, Capitani E, Della Sala S. MODA. Milan Overall Dementia Assessment: Manual. Firenze: Giunti OS; 1994.

20. Katz S. Assessing self-maintenance: activities of daily living, mobility, and instrumental activities of daily living. $\mathrm{J} \mathrm{Am} \mathrm{Geriatr} \mathrm{Soc.}$ 1983;31(12):721-727.

21. Lawton MP, Brody EM. Instrumental activities of daily living scale (IADL). Gerontologist. 1969;9:179-186.

22. Hoehn MM, Yahr MD. Parkinsonism: onset, progression, and mortality. 1967. Neurology. 1998;50(2):318-334.

23. Goetz CG, Tilley BC, Shaftman SR, et al. Movement disorder societysponsored revision of the unified Parkinson's disease rating scale (MDS-UPDRS): scale presentation and clinimetric testing results. Mov Disord. 2008;23(15):2129-2170.

24. Yesavage JA, Brink TL, Rose TL, et al. Development and validation of a geriatric depression screening scale: a preliminary report. J Psychiatr Res. 1982;17(1):37-49.

25. Brazzelli M, della Sala S, Laiacona M. Taratura della versione italiana dei Rivermead Behavioral Memory Test: un test di valutazione ecologica della memoria [Calibration of the Italian version of the Rivermead Behavioral Memory Test: an ecological evaluation of memory]. Bollettino di psicologia applicata. 1993;206:33-42. Italian.

26. Dubois B, Slachevsky A, Litvan I, Pillon B. The FAB: a Frontal assessment battery at bedside. Neurology. 2000;55(11):1621-1626.

27. Appollonio I, Leone M, Isella V, et al. The Frontal Assessment Battery (FAB): normative values in an Italian population sample. Neurol Sci. 2005;26(2):108-116.

28. Iavarone A, Ronga B, Pellegrino L, et al. The Frontal Assessment Battery (FAB): normative data from an Italian sample and performances of patients with Alzheimer's disease and frontotemporal dementia. Funct Neurol. 2004;19(3):191-196.

29. Bhidayasiri R, Tarsy D. Movement Disorders: A Video Atlas. New York: Humana Press; 2012.

30. Foster ER, Bedekar M, Tickle-Degnen L. Systematic review of the effectiveness of occupational therapy-related interventions for people with Parkinson's disease. Am J Occup Ther. 2014;68(1):39-49.

31. Oosterveld LP, Allen JC, Reinoso G, et al. Prognostic factors for early mortality in Parkinson's disease. Parkinsonism Relat Disord. 2015; 21(3):226-230.

32. Uysal-Cantürk P, Hanağası HA, Bilgiç B, Gürvit H, Emre M. An assessment of Movement Disorder Society Task Force diagnostic criteria for mild cognitive impairment in Parkinson's disease. Eur J Neurol. 2018; 25(1):148-153.
33. Sveinbjornsdottir S. The clinical symptoms of Parkinson's disease. J Neurochem. 2016;139(Suppl 1):318-324.

34. Zhong JY, Moffat SD. Age-related differences in associative learning of landmarks and heading directions in a virtual navigation task. Front Aging Neurosci. 2016;8:122.

35. Benke T, Karner E, Petermichl S, Prantner V, Kemmler G. Neuropsychological deficits associated with route learning in Alzheimer disease, MCI, and normal aging. Alzheimer Dis Assoc Disord. 2014;28(2):162-167.

36. Burgess N. Spatial cognition and the brain. Ann N Y Acad Sci. 2008;1124(1):77-97.

37. Doeller CF, Burgess N. Distinct error-correcting and incidental learning of location relative to landmarks and boundaries. Proc Natl Acad Sci US A. 2008;105(15):5909-5914.

38. Doeller CF, King JA, Burgess N. Parallel striatal and hippocampal systems for landmarks and boundaries in spatial memory. Proc Natl Acad Sci U S A. 2008;105(15):5915-5920.

39. Davidsdottir S, Wagenaar R, Young D, Cronin-Golomb A. Impact of optic flow perception and egocentric coordinates on veering in Parkinson's disease. Brain. 2008;131(11):2882-2893.

40. Lord S, Archibald N, Mosimann U, Burn D, Rochester L. Dorsal rather than ventral visual pathways discriminate freezing status in Parkinson's disease. Parkinsonism Relat Disord. 2012;18(10):1094-1096.

41. Davidsdottir S, Wagenaar R, Young D, Cronin-Golomb A. Impact of optic flow perception and egocentric coordinates on veering in Parkinson's disease. Brain. 2008;131(Pt 11):2882-2893.

42. Hanna-Pladdy B, Pahwa R, Lyons KE. Paradoxical effect of dopamine medication on cognition in Parkinson's disease: relationship to side of motor onset. JINS. 2015;21(4):259-270.

43. Pirogovsky-Turk E, Filoteo JV, Litvan I, Harrington DL. Structural MRI correlates of episodic memory processes in Parkinson's disease without mild cognitive impairment. J Parkinsons Dis. 2015;5(4):971-981.

44. Duff K, Schoenberg MR, Scott JG, Adams RL. The relationship between executive functioning and verbal and visual learning and memory. Arch Clin Neuropsychol. 2005;20(1):111-122.

45. Takagi R, Kajimoto Y, Kamiyoshi S, Miwa H, Kondo T. The frontal assessment battery at bed side (FAB) in patients with Parkinson's disease. No To Shinkei. 2002;54(10):897-902.

46. Matsui H, Udaka F, Miyoshi T, et al. Frontal assessment battery and brain perfusion image in Parkinson's disease. J Geriatr Psychiatry Neurol. 2006;19(1):41-45.

47. Lima CF, Meireles LP, Fonseca R, Castro SL, Garrett C. The frontal assessment battery (FAB) in Parkinson's disease and correlations with formal measures of executive functioning. J Neurol. 2008;255(11):1756-1761.

48. Latt MD, Lord SR, Morris JG, Fung VS. Clinical and physiological assessments for elucidating falls risk in Parkinson's disease. Mov Disord. 2009;24(9):1280-1289.

49. Robinson H, Calamia M, Gläscher J, Bruss J, Tranel D. Neuroanatomical correlates of executive functions: a neuropsychological approach using the EXAMINER battery. J Int Neuropsychol Soc. 2014;20(1): $52-63$.

50. van der Linden M, Meulemans T, Marczewski P, et al. The relationships between episodic memory, working memory, and executive functions: the contribution of the prefrontal cortex. Psychol Belg. 2000;40(4):275-297.

51. Bezdicek O, Růžička F, Fendrych Mazancova A, et al. Frontal Assessment Battery in Parkinson's disease: validity and morphological correlates. J Int Neuropsychol Soc. 2017;23(8):675-684.

52. Armstrong RA. Visual symptoms in Parkinson's disease. Parkinsons Dis. Epub 2011 May 25.

53. Pawlitzki E, Schlenstedt C, Schmidt N, et al. Spatial orientation and postural control in patients with Parkinson's disease. Gait Posture. 2018; 60:50-54

54. Hanna-Pladdy B, Pahwa R, Lyons KE. Paradoxical effect of dopamine medication on cognition in Parkinson's disease: relationship to side of motor onset. J Int Neuropsychol Soc. 2015;21(4):259-270. 
55. Tu S, Spiers HJ, Hodges JR, Piguet O, Hornberger M. Egocentric versus allocentric spatial memory in behavioral variant frontotemporal dementia and Alzheimer's disease. J Alzheimers Dis. 2017;59(3):883-892.

56. Kudlicka A, Clare L, Hindle JV. Executive functions in Parkinson's disease: systematic review and meta-analysis. Mov Disord. 2011; 26(13):2305-2315.
57. Peña J, Ibarretxe-Bilbao N, García-Gorostiaga I, Gomez-Beldarrain MA, Díez-Cirarda M, Ojeda N. Improving functional disability and cognition in Parkinson disease: randomized controlled trial. Neurology. 2014;83(23):2167-2174.

\section{Publish your work in this journal}

Neuropsychiatric Disease and Treatment is an international, peerreviewed journal of clinical therapeutics and pharmacology focusing on concise rapid reporting of clinical or pre-clinical studies on a range of neuropsychiatric and neurological disorders. This journal is indexed on PubMed Central, the 'PsycINFO' database and CAS, and is the official journal of The International Neuropsychiatric Association (INA). The manuscript management system is completely online and includes a very quick and fair peer-review system, which is all easy to use. Visit http://www.dovepress.com/testimonials.php to read real quotes from published authors.

Submit your manuscript here: http://www.dovepress.com/neuropsychiatric-disease-and-treatment-journal 